\title{
Amyloid Myoneuropathy Mimicking Inclusion Body Myositis
}

\author{
Ahmad R. Abuzinadah, Jeffrey T. Joseph, Lawrence Korngut
}

Can J Neurol Sci. 2013; 40: 255-258

Inclusion body myositis (IBM) is a slowly progressive myopathy affecting proximal and distal musculature with typical onset of symptoms after age 50 years. Inclusion body myositis more often affects males and results in a classical pattern of early asymmetric weakness and wasting of forearm flexors (wrist and long finger flexors), quadriceps and ankle dorsiflexors. Inclusion body myositis is more frequently associated with a monoclonal gammopathy than is seen in the general population but the implications of this association remain unclear ${ }^{1}$. Importantly, there is no available therapy to slow disease progression in IBM. Primary systemic amyloidosis (AL) typically results in light chain deposition in the tongue, kidneys, heart, liver, spleen and peripheral nerves with accompanying respective clinical sequelae. Rarely, light chain deposition can focally involve components of the nervous system. Peripheral nerve complications include carpal tunnel syndrome, dysautonomia and polyneuropathy ${ }^{2,3}$. Amyloid myopathy is rare and typically demonstrates proximal muscle involvement and electrophysiological findings indistinguishable from the inflammatory myopathies ${ }^{4}$. Prompt differentiation of amyloid myopathy from the inflammatory myopathies is essential as AL may respond to chemotherapy resulting in improved progression free survival ${ }^{5}$. We report a case of AL presenting with predominant myopathy clinically suggestive of IBM and with similar findings on initial muscle biopsy, an important distinction in light of recent treatment advances in AL.

\section{Case Report}

A 59-year-old right-handed man was referred to the Calgary Neuromuscular Clinic for progressive weakness over five years. He described left knee "give-way" while skiing as his initial complaint. Over time, he noticed increasing difficulty to keep up with others while walking. Ascending stairs became more challenging and his legs would give away intermittently. He denied symptoms of foot drop or proximal limb weakness. Gradually he noticed progressive bilateral hand grip weakness associated with dropping of items. He was still able to button up clothing and use a zipper. Over the past year he observed that his ability to reach up with his hands became more difficult and that food would stick in his throat without choking. Since the onset of weakness the patient described shortness of breath on exertion, and more recently, orthopnea but no morning headache. The patient did not have any cognitive complaints, rash, sensory symptoms, pain, diplopia or ptosis. His past medical history and family history were non-contributing to his final diagnosis.

On examination cardiac, abdominal and respiratory examinations were normal. Mental status, language testing and cranial nerve examination were normal. No facial weakness, macroglossia or tongue fasciculations were observed.

Motor examination revealed no wasting, fasciculations, pseudohypertrophy, or scapular winging. Tone was normal. Neck flexion and extension were full power. Weakness was noted as follows: deltoids 4+, biceps 4+, flexor pollicis longus 5-, flexor digitorum profundus 4 in the index and middle fingers and 5 in ring and little fingers bilaterally, hip flexors 2-3, knee extension 4 (left) and 5- (right), knee flexion 4 . The remainder of the detailed muscle power examination including ankle dorsiflexion was normal. Mild hyporeflexia was observed throughout but most prominently at the left patella. No lower extremity edema was observed. Pinprick and temperature exam showed reduced sensation up to mid arms and legs bilaterally. Proprioception was normal. Cerebellar testing was normal. Gait was waddling and not ataxic.

Investigations prior to the clinic appointment included normal CBC, lytes, creatinine, urea, magnesium, phosphate, calcium, ESR, C-reactive protein, total bilirubin, ALP and lipase. Elevated creatine kinase 431 (reference range (RR): 0$195 \mathrm{U} / \mathrm{L}$ ), lactate dehydrogenase 268 (RR: 100-235 U/L), ALT 71 (RR:1-60) and GGT 104 (RR:11-63) were noted. Twentyfour hour urine protein was $0.3 \mathrm{~g}$ (RR: $0.0-0.15 \mathrm{~g} / 24 \mathrm{hrs}$ ), elevated but not in the nephrotic range. ANA was positive at 1:160 and ENA were negative. Serum protein electrophoresis demonstrated an IgG Kappa monoclonal gammopathy (2.9 g/L), and urine protein electrophoresis showed free kappa light chains consistent, on Hematologic consultation, with a monoclonal gammopathy of unknown significance or smoldering myeloma not requiring intervention. On previous work up by respirology intermittent lower limb swelling was reported. He had no symptoms at rest and no chest pain, cough, weight loss, fever, anorexia. Computed tomogram (CT) chest, cardiac echo and angiogram were unremarkable. His pulmonary function tests revealed reduced residual volume of $69 \%$ (Normal: $>80 \%$ ) and a $15 \%$ reduction in forced expiratory volume (FEV1) in supine compared to sitting positions. The respirologist ruled out

From the Department of Clinical Neuroscience (ARA, LK), Hotchkiss Brain Institute, Department of Pathology (JTJ), University of Calgary, South Health Campus, Calgary, Alberta, Canada.

Received June 4, 2012. Final Revisions Submitted September 5, 2012. Correspondence to: Lawrence Korngut, 4th Floor Administration, Clinical Neurosciences, South Health Campus, 4448 Front Street S.E., Calgary, Alberta, T3M 1M4, Canada.Email: korngut@gmail.com. 


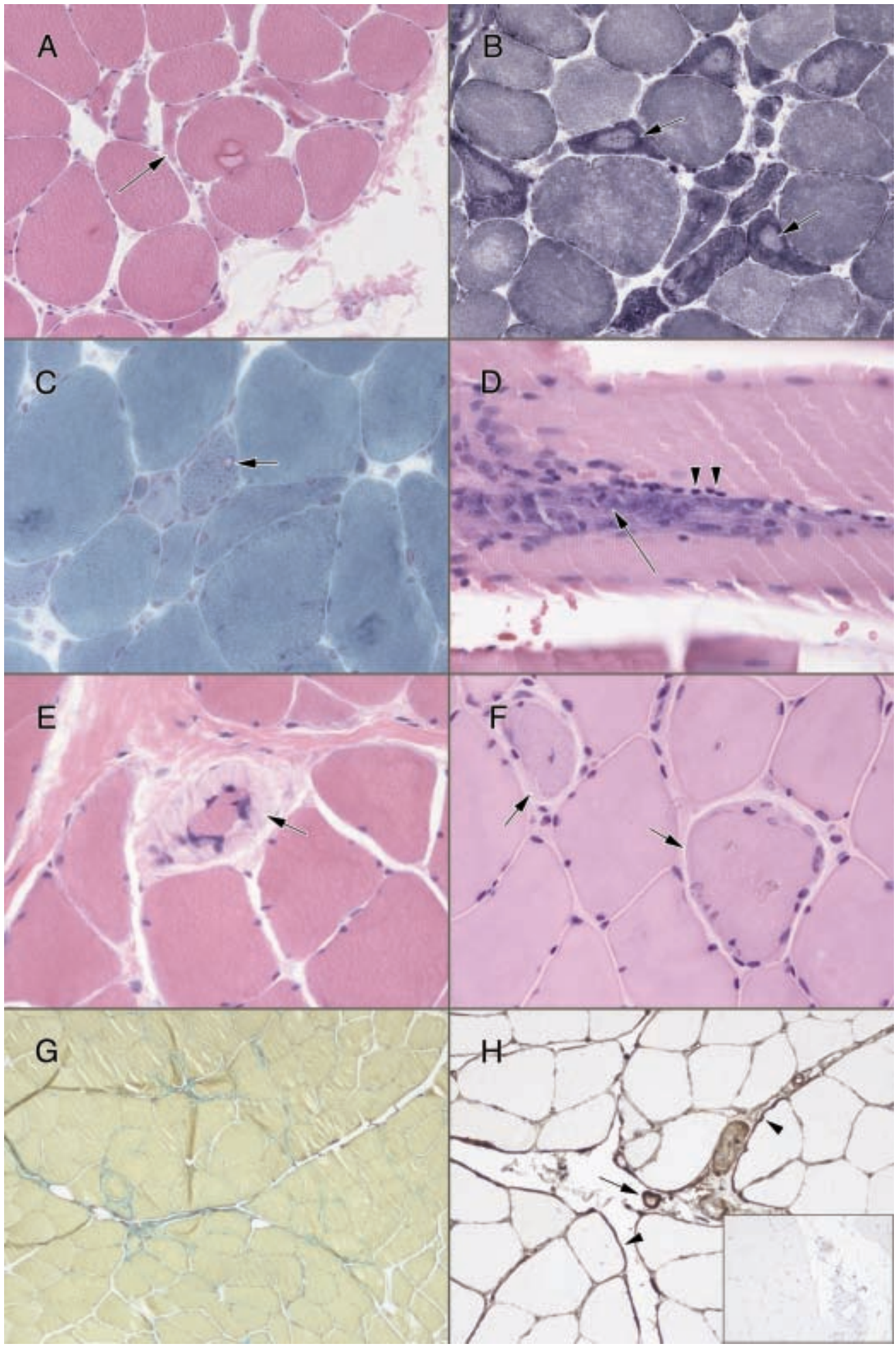

Figure: Muscle pathology. The panels illustrate the earlier left vastus lateralis muscle biopsy (panels A - D) and later left biceps muscle biopsy (panels $E-H)$. Panel $D$ is from formalin fixed, paraffin embedded tissue, while the remainder are from snap-frozen muscle. Panel A is a hematoxylin-eosin stain that demonstrates the small group atrophy and small, angulated myofibers (arrow) typical of denervation changes (original magnification 20x). NADH histochemistry on a similar cluster (panel B) revealed frequent target and targetoid fibers (arrows) (original magnification 20x). Only rare rimmed vacuoles were present in Gomori trichrome staining (panel C; original magnification 20x). The muscle contained scattered necrotic myofibers (panel D) that were replaced by macrophages (arrow) and has scant lymphocytic inflammation (arrowheads; original magnification 40x). The second biopsy had prominent amorphous material around individual myofibers (panels $E \& F$, arrow; original magnification 40x). The sulphated Alcian blue (SAB) stain for amyloid showed this material to be positive (panel $G$, green staining; original magnification 10x). Panel H showed the material immunoreacts for kappa light chain, both around vessels (arrow) and around individual myofibers (arrowheads; original magnification 20x). The inset in panel H shows no significant immunoreactivity for lambda light chain (original magnification 20x). 
restrictive or obstructive lung disease and questioned possible respiratory muscle weakness.

Recent nerve conduction studies performed elsewhere had revealed an absent sural sensory response. Electromyography (EMG) had revealed frequent fibrillations and positive sharp waves in iliopsoas and rare discharges in tibialis anterior. Early recruitment was noted in deltoid and iliopsoas superimposed upon reduced recruitment in tibialis anterior, vastus medialis and first dorsal interosseous. Increased insertional activity, mild to moderate neurogenic motor unit remodeling and reduced interference pattern with mildly increased firing rate were observed in all muscles sampled except triceps and extensor digitorum communis which were both normal in all regards. The study was interpreted as a proximal myopathy, likely inflammatory, with a superimposed mild diffuse length dependent axonal sensory and motor polyneuropathy.

A left vastus lateralis muscle biopsy identified a myopathy with two components: denervation-reinnervation changes (small group atrophy - panel A, targetoid fibers - panel B) and a primary myopathy (a few rimmed vacuoles - panel $\mathrm{C}$, necrotic and regenerating fibers, predominantly macrophage inflammation - panel D). TDP-43 and ubiquitin immunostains were normal.

A working diagnosis of IBM was made based upon the pattern of weakness (prominent long finger flexors and knee extensor involvement), mixed myogenic and neurogenic electrophysiological findings, and the myopathic muscle biopsy findings associated with mild inflammation and very rare but present rimmed vacuoles.

Myositis-related antibodies were dispatched and were all negative including Mi-2, Ku, PM-Scl, Jo-1, PL-7, PL-12, and Ro 52. Cryoglobulins were negative.

Nine months later the patient returned for follow-up. The interval history revealed that his left knee now gave away on a daily basis, his gait was slower and he was experiencing more frequent falls. He also had persistent lower extremity swelling and increased shortness of breath. His symptoms otherwise remained stable. The prior muscle biopsy was reviewed by neuropathology and no staining with either Kappa or Lambda light chains was observed. The patient's shortness of breath worsened further and he was diagnosed with congestive heart failure on chest X-ray. CT chest, abdomen and pelvis showed mild bilateral pleural effusion.

Nerve conduction studies were repeated in the right arm and leg and revealed normal sensory studies except for a moderately reduced sural amplitude. Motor nerve conduction studies were essentially normal. Electromyography demonstrated very prominent positive sharp waves and fibrillation potentials in the deltoid, biceps, iliopsoas, vastus lateralis and tibialis anterior muscles. Early recruitment of myopathic units was seen in deltoid, biceps and iliopsoas with reduced recruitment of rapid firing neurogenic motor units observed in the right extensor indicis proprius and vastus lateralis.

A repeat echocardiogram demonstrated left ventricular dysfunction. Cardiac MRI revealed a minimally reduced ejection fraction (49\%; normal $>50 \%$ ) and findings consistent with an infiltrative process. Due to concern for AL it was decided to proceed with a repeat muscle biopsy.
A left biceps muscle biopsy showed amorphous material deposited in vessel walls (panel E), around the border of the perifascicular region, and into the endomysium (panel F). This material stained for Congo red and showed faint green birefringence under polarized light. It stained strongly for the amyloid stain sulphated alcian blue (SAB; panel G). Immunostaining demonstrated specific immunoreactivity of the amorphous material for kappa light chain antibodies (panel $\mathrm{H}$ ) but not for lambda light chain (panel $\mathrm{H}$ inset).

Hematology agreed with the diagnosis of AL amyloidosis and treatment with dexamethasone and melphalan were initiated. Five months after diagnosis he was re-admitted for congestive heart failure complicated by pleural effusion and bacterial pneumonia. He was treated with aggressive diuresis and antibiotics. His symptoms improved and he was ambulating which lead to stopping his DVT prophylaxis. One day prior to planned discharge he developed acute onset shortness of breath and pulseless electrical activity arrest. The patient died from a presumed pulmonary embolism. Autopsy was declined by his family.

\section{Discussion}

Our patient presented with later-onset gradually progressive weakness with an asymmetric distal-proximal pattern on examination suggestive of IBM. Electromyography provided confirmation of a myopathy with active spontaneous discharges suggesting inflammation and superimposed chronic motor unit denervation. Prominent hip flexion weakness is atypical but does not exclude IBM. As cited in this case report the patient had an absent sural sensory response on nerve conduction studies consistent with an axonal polyneuropathy that is often seen in $\mathrm{IBM}^{6}$. The initial muscle biopsy demonstrated features suggestive of IBM, including rimmed vacuoles. While rimmed vacuoles are helpful in the diagnosis of IBM they are neither sensitive nor specific as the differential diagnosis includes dermatomyositis, the myofibrillar myopathies and denervation ${ }^{7,8}$. On follow-up, heart failure and prominent pedal edema were observed. The initial muscle biopsy was rereviewed and deposition of light chains was sought but not found. The combination of new heart failure, rapid progression of weakness and IgG Kappa monoclonal gammopathy prompted a second muscle biopsy that demonstrated kappa light chain deposition and confirmed the diagnosis of AL amyloidosis. The possibility of dual diagnosis (i.e IBM and amyloid myopathy) was felt to be unlikely given the presence of paraproteinemia on initial presentation and rapid progression of weakness.

In retrospect, the clinical finding of hip flexor greater than knee extensor weakness was atypical for IBM. Predominant proximal weakness and isolated hip flexor weakness has been reported previously with amyloid myopathy ${ }^{9-11}$. The early features suggestive of amyloidosis were the presence of a light chain monoclonal gammopathy, urine light chains and recurrent pedal edema. The initial muscle biopsy revealed features suggestive of IBM with no evidence of amyloid deposition on subsequent re-staining for light chains. Most often amyloid myopathy presents with proximal muscle weakness but distal muscle weakness is observed in $10 \%$ of patients and absence of classic stigmata of amyloidosis like macroglossia and muscular pseudohypertrophy can be seen up to $9 \%^{3}$. Amyloid myopathy 
with rimmed vacuoles mimicking IBM has only been reported once previously ${ }^{12}$. In one study, monoclonal gammopathies of unknown significance were found in $22.8 \%$ of IBM patients including Kappa and Lambda light chains ${ }^{1}$. Such monoclonal proteins have been demonstrated to convert to lymphoproliferative disorders in a rate of $1 \%$ per year ${ }^{13}$.

$\mathrm{AL}$ can be difficult to diagnose even with a high index of suspicion. Sensitivity of SPEP in amyloidosis is only 50\%. SPEP can identify up to $90 \%$ of cases when combined with immunofixation but it is often unavailable. Amyloid myopathy can be even more difficult to confirm and bone marrow biopsy may be negative in $90 \%$ of patients ${ }^{3}$. Electrophysiological examination commonly demonstrates evidence of active denervation in the form of positive sharp waves and fibrillation potentials on EMG in patients with IBM and amyloid myopathy ${ }^{3}$.

Muscle biopsy in amyloid myopathy may not show amyloid deposition in the initial biopsy and repeat biopsy is needed in $24 \%$ of the cases ${ }^{3}$. Moreover, amyloid myopathy may show inflammatory infiltrates on muscle biopsy that lead to misdiagnosis of polymyositis in $5 \%$ of cases $^{3}$. This case also demonstrates that amyloid myopathy may be associated with rimmed vacuoles.

This case highlights the close similarity between IBM and amyloid myoneuropathy in regards to clinical, laboratory and electrophysiological findings. The diagnosis of amyloidosis should be considered on the differential of distal myopathies. The presence of rapid progression, heart failure, leg edema and monoclonal gammopathy should prompt consideration of additional investigations including repeat muscle biopsy. Recent studies have demonstrated an effective therapy for early AL amyloidosis which emphasizes the need for prompt diagnosis ${ }^{5}$.

\section{REFERENCES}

1. Dalakas MC, Illa I, Gallardo E, Juarez C. Inclusion body myositis and paraproteinemia: incidence and immunopathologic correlations. Ann Neurol. 1997;41(1):100-4.

2. Matsuda M, Gono T, Morita H, Katoh N, Kodaira M, Ikeda S. Peripheral nerve involvement in primary systemic AL amyloidosis: a clinical and electrophysiological study. Eur J Neurol. 2011;18(4):604-10.

3. Chapin JE, Kornfeld M, Harris A. Amyloid myopathy: Characteristic features of a still underdiagnosed disease. Muscle Nerve. $2005 ; 31(2): 266-72$.

4. Rubin DI, Hermann RC. Electrophysiologic findings in amyloid myopathy. Muscle Nerve. 1999;22(3):355-9.

5. Venner CP, Lane T, Foard D, et al. Cyclophosphamide, bortezomib, and dexamethasone therapy in AL amyloidosis is associated with high clonal response rates and prolonged progression-free survival. Blood. 2012;119(19):4387-90.

6. Hermanns B, Molnar M, Schröder JM. Peripheral neuropathy associated with hereditary and sporadic inclusion body myositis: confirmation by electron microscopy and morphometry. J Neurol Sci. 2000;179(1-2):92-102.

7. Schoser B. Physiology, pathophysiology and diagnostic significance of autopahgic changes in skeletal muscle tissue towards the enigma of rimmed and round vacules. Clin Neuropathol. 2009;28(1):59-70.

8. Limaye $\mathrm{V}$, Blumbergs $\mathrm{P}$. The prevalence of rimmed vacuoles in biopsy-proven dermatomyositis. Muscle Nerve. 2010;41(2): 288-9.

9. Bruni J, Bilbao JM, Pritzker PH. Myopathy associated with amyloid angiopathy. Can J Neurol Sci. 1977;4(1):77-80.

10. Roke ME, Brown WF, Boughner D, Ang LC, Rice GP. Myopathy in primary systemic amyloidosis. Can J Neurol Sci. 1988;15(3): 314-6.

11. Keith J, Afshar-Ghotli Z, Roussev R, Ernst B, Young B, Bilbao J. Myopathy as the Initial Manifestation of Primary Amyloidosis. Can J Neurol Sci. 2011;38(1):161-4.

12. Tuomaala H, Kärppä M, Tuominen H, Remes AM. Amyloid myopathy: a diagnostic challenge. Neurol Int. 2009;1(e7):24-6.

13. Kyle RA, Therneau TM, Rajkumar SV, et al. A long-term study of prognosis in monoclonal gammopathy of undetermined significance. N Engl J Med. 2002;346(8):564-9. 